\title{
Apuntes en sismología e instrumentación
}

Sebastián Soto G.*

\section{Resumen}

En Colombia la probabilidad de eventos sísmicos y vulcanológicos es alta debido a que en el territorio interactúan tres grandes placas tectónicas: la de Nazca, la suramericana y la del Caribe. Su existencia originó la formación de grandes fallas geológicas a lo largo del país y desencadenó una actividad sísmica frecuente en regiones del altiplano cundiboyacense, el eje cafetero y los Santanderes. En el presente artículo de revisión se presentan conceptos básicos de sismología, los aspectos más importantes para considerar un sismo y sus características, desarrollos en instrumentación para el campo de monitoreo de amenazas sísmicas y la prevención de eventos de este tipo.

Palabras clave: amenaza sísmica, instrumentos de monitoreo sismológico, prevención de desastres, sismología

\begin{abstract}
Colombia has a high probability of seismic and volcanological events, due to the interaction of three large tectonic plates: the Nazca plate, the South American plate and the Caribbean plate. Their existence originated the formation of large geological faults throughout the country and triggered frequent seismic activity in regions of Cundiboyacense high plateau, the coffee axis and Santanderes. This review article presents basic concepts of seismology, the most important aspects to consider an earthquake and its characteristics, developments in instrumentation for the field of seismic hazard monitoring and the prevention of events of this type.
\end{abstract}

Keywords: Disaster Prevention, Seismic Monitoring Instruments, Seismic Threat, Seismology 


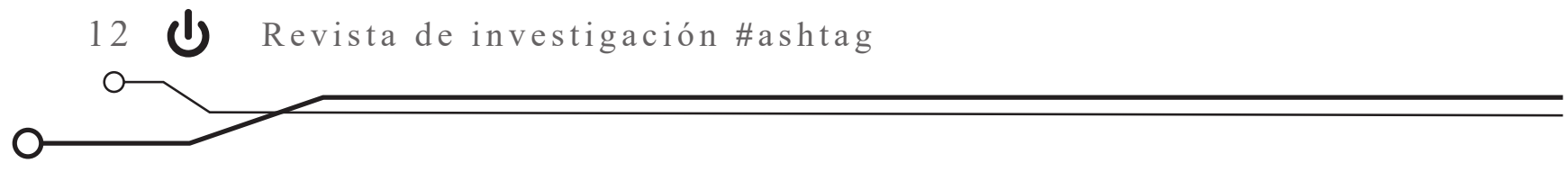

\section{Contexto sísmico en Colombia y el mundo}

Los sismos o terremotos son originados por vibraciones de la tierra que liberan una gran cantidad de energía en forma de ondas. Actualmente se producen alrededor de 300000 sismos perceptibles de los cuales 75 son realmente significativos. La mayoría ocurren en lugares remotos; sin embargo, un porcentaje de estos acaecen cerca a zonas pobladas, lo que convierte a este fenómeno en la fuerza más destructiva de la naturaleza. El temblor del terreno, junto con la licuefacción de algunos sólidos, siembra la devastación en edificios y otras estructuras. Además, cuando se produce un terremoto en un área poblada, suelen romperse las tuberías de gas y líneas de energía, principal causa de los incendios durante estos movimientos telúricos (Tarbuck y Lutgens, 2005).

La historia del planeta Tierra ha tenido una estrecha relación con los sismos: entre ellos, se encuentra el terremoto de Portugal del año 1755, de magnitud 8.7 grados en la escala de Richter de magnitud de momento $\left(\mathrm{M}_{\mathrm{w}}\right)$. Agrietó la tierra, género un enorme tsunami de 10 metros de altura y causó la muerte a 100000 personas aproximadamente entre Portugal, España y Marruecos. También se tiene registro del gran terremoto de Chile del año 1960, de magnitud 9.5 en la escala Magnitud de momento $\left(\mathrm{M}_{\mathrm{w}}\right)$. Ha sido el terremoto más grande y potente jamás registrado. El sismo fue percibido en diferentes partes del planeta y produjo un tsunami que viajó por todo el Pacífico hasta Japón y Hawái, donde más de 200 personas fallecieron. Solo en Chile dos millones de personas perdieron su hogar. Se estima que esta catástrofe natural dejó entre 1665 y 2000 muertos, así como innumerables daños materiales.

El terremoto de Boxing Day del año 2004, de magnitud 9.1 en la escala $M_{w^{\prime}}$ cambió la vida de millones de personas en algunos de los países más pobres del mundo. Este terremoto ha sido el tercero más intenso registrado y el segundo con el mayor saldo de muertes. Además, ocasionó una serie de tsunamis devastadores a lo largo de las costas de la mayoría de los países que bordean el océano Índico que provocó grandes inundaciones en Indonesia, Malasia, Sri Lanka, la India y Tailandia. El número de víctimas superó los 220000 y 1,7 millones de personas tuvieron que ser evacuadas.

En el año 2010, el terremoto de Haití dejó la cifra de 316000 muertos; el más devastador del siglo XXI hasta el momento. El epicentro fue registrado en su capital, Puerto Príncipe, con una magnitud de 7.2 grados en la escala ML. Este fue seguido de varias réplicas de 5.9, 5.5 y 5.1 grados ( $B B C$ Mundo, 13 de enero del 2010). Para el año 2011, un terremoto de magnitud 9.0 en la escala $M_{w}$ se convirtió en el más fuerte que ha sufrido Japón hasta la actualidad, con 15883 muertos y 2650 desaparecidos. Además de la catástrofe, se declaró un estado de emergencia en la central nuclear de Fukushima debido al fallo de los sistemas de refrigeración de uno de los reactores. Después de una explosión en la central, las autoridades evacuaron a 45000 personas por peligro de radiación nuclear, lo que hizo de este el incidente el más grave desde Chernóbil (Gómez, 18 de septiembre del 2017).

Colombia está situada en el cinturón de fuego del Pacífico, por lo tanto, el país es vulnerable a sismos de gran magnitud, como los registrados en Cúcuta en 1875, que dejaron la ciudad semi destruida y con un saldo de mil muertos. En 1979, el segundo sismo más fuerte del siglo en Colombia destruyó la población de San José de la Costa y parcialmente a El Charco, Tumaco y 
otras poblaciones. En 1983, el terremoto de Popayán dejó 300 muertos y 508 heridos. El 6 de junio de 1994 un terremoto causó la avalancha del río Páez, en el departamento del Cauca, y dejó 800 muertos y 55000 indígenas damnificados (El Tiempo, 25 de enero de 1999).

La sismología surgió para responder al peligro que representan los terremotos. Tiene por objetivos principales estudiar la propagación de las ondas sísmicas por el interior de la tierra, determinar las causas que dan origen a los temblores

\section{Historia de la sismología en Colombia}

En 1940, el padre Jesús Emilio Ramírez regresó al país después de presentar su tesis doctoral en la Universidad de Saint Louis, Estados Unidos, en la que planteó la posibilidad de detectar los huracanes con los sismógrafos. Poco tiempo después, decidió fundar el Instituto Geofísico de los Andes Colombianos, cuya aparición fue un hecho muy importante a nivel continental y el primer paso para la sismología el país.

El Instituto Geofísico de los Andes Colombianos pasó a tener cinco estaciones sismológicas. $\mathrm{Su}$ red registraba los movimientos in situ, en un papel que debía ser llevado a Bogotá junto con los demás registros para su respectivo análisis. Esto conllevaba que los análisis se efectuaran días después de los eventos sísmicos. Otro problema de la red era la desincronización de los equipos respecto del tiempo internacional, lo que averiaba el marcador del sismógrafo y sacaba al equipo de la red hasta que se gestionará su reposición, que podía tardar hasta 6 meses.

Tiempo después, la Asociación Colombiana de Ingeniería Sísmica comenzó a trabajar para y alertar a la población sobre posibles sismos. Los sismógrafos tienen un papel fundamental en el registro y examen de los fenómenos naturales; con base en ellos, pueden analizar e interpretar el paso de las ondas sísmicas según su tipo. El registro y acumulación de los sismogramas permiten determinar el grado de sismicidad de una región. Conjuntamente, el constante monitoreo de los sismos puede llegar a determinar la frecuencia con la que ocurre un terremoto y las posibles afectaciones sobre la población (Castro et al. 2008).

construir una red más moderna. El Instituto Geofísico de la Universidad Javeriana contaba con cinco acelerógrafos donados por el Servicio Geológico de los Estados Unidos, que dieron origen a una nueva red.

Entre 1987 y 1993 iniciaron las obras civiles para la recepción de análisis de datos en la ciudad de Bogotá, además de la construcción de cada una de las estaciones. La capacitación del personal fue impartida en Canadá en 1988. Para finales de 1993, se organizó el funcionamiento de las primeras 13 estaciones. Los siguientes años de la década de los noventa fueron muy importantes para la red. En 1994 se inauguró oficialmente la Red Sismológica Nacional de Colombia (RSNC). Entre 1995 y 1997 la donación del Gobierno central permitió ampliar la red a 20 estaciones sismológicas y 50 estaciones acelerográficas. En ese mismo año, la Agencia de Cooperación Internacional del Japón (JICA) donó a la RSNC un sistema automático de localización de sismos y empezó a funcionar la estación ALPHA, ubicada en El Rosal, Cundinamarca (Servicio Geológico Colombiano, 2017). 


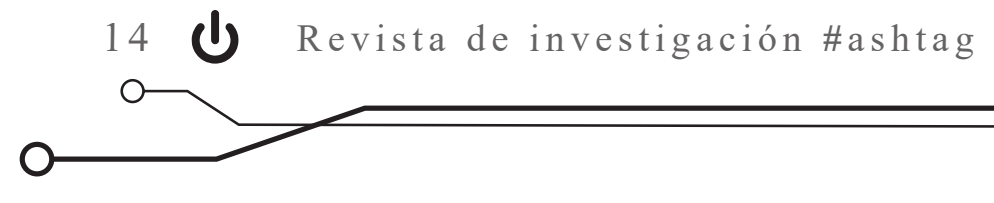

En la actualidad, la red cuenta con 62 estaciones sismológicas que transmiten en tiempo real en todo el territorio nacional. Esta cuenta, además, con 122 estaciones acelerográficas, de las cuales

\section{Características de un sismo}

\section{Terremoto}

Un terremoto es la vibración de la Tierra producida por una rápida liberación de energía a causa del deslizamiento de la corteza terrestre a lo largo de una falla. La energía liberada se propaga en todas las direcciones desde su origen (foco o hipocentro) en forma de ondas exactamente iguales a las producidas al lanzar una piedra en un estanque. El terremoto produce ondas sísmicas que se propagan en la Tierra. Aunque la energía liberada por la "ruptura" (las ondas) se disipa rápidamente al alejarse del foco, es posible registrar el movimiento en cualquier punto de la Tierra por medio de aparatos suficientemente sensibles (Tarbuck y Lutgens, 2005).

\section{Ruptura y propagación de un terremoto}

La ruptura inicial comienza en el foco y se propaga alejándose del punto de origen, algunas veces en dos direcciones horizontales a lo largo de la falla. De acuerdo con un modelo, el deslizamiento en cualquier lugar a lo largo de una falla se logra de manera casi instantánea. Además, en cualquier momento, el deslizamiento se limita a tan solo una zona estrecha a lo largo de la falla, que se desplaza hacia delante de manera continua. A medida que esta zona de ruptura avanza, puede reducir su velocidad, acelerar o incluso saltar a un segmento cercano de falla.
45 transmiten en tiempo real y las demás deben ser visitadas para descargar los datos (Servicio Geológico Colombiano, 2018).

En la corteza de la tierra se acumula energía debido a procesos de deformación elástica, definidos como la rotura de las rocas después de que es superado el límite elástico. El rebote elástico es similar a lo que sucede al doblar una varilla de madera: se supera la resistencia friccional que mantiene unidas las rocas $\mathrm{y}$, a medida que se produce el deslizamiento en los puntos más débiles (el foco), el desplazamiento provoca un aumento de los esfuerzos en las zonas más alejadas a lo largo de la falla, donde un nuevo desplazamiento liberará la mayor parte de la energía elástica acumulada. Este deslizamiento permite que la roca regrese a su posición de partida (Tarbuck y Lutgens, 2005).

En los terremotos de baja intensidad, el deslizamiento se produce a lo largo de la falla comparativamente pequeña; de esta manera, puede propagarse rápidamente y el tiempo del sismo es relativamente corto, a diferencia de los sismos de grandes magnitudes, generados por un desplazamiento a lo largo de una falla que puede medir unos cientos de kilómetros (Tarbuck y Lutgens, 2005). 


\section{Ondas sísmicas}

Las ondas sísmicas son producidas por la liberación de energía mecánica y son de tipo elástico. En el proceso de la fuente sísmica, son las encargadas de transportar la energía desde el foco a la superficie y son generadas por movimientos

\section{Localización de los sismos}

El hipocentro o foco es la zona en el interior de la Tierra donde inicia la ruptura de la falla; desde ahí se propagan las ondas sísmicas. El epicentro es el punto en la superficie terrestre situado directamente encima del hipocentro. Este último está dado por 4 valores (tiempo, latitud, longitud y profundidad donde se inicia la liberación de energía) y se determina por los tiempos de llegada de cada una de las ondas a los sismómetros que están alrededor del evento sísmico. Para esto es necesaria la identificación de las fases sísmicas y la elección del modelo terrestre.

\section{Amenaza sísmica en Colombia}

Colombia es uno de los países que se encuentran ubicados en lo que se denomina el cinturón de fuego del Pacífico, a saber, la zona de mayor actividad sísmica y vulcanológica del planeta, debido a que tiene las áreas de subducción más importantes del mundo. El territorio colombiano se encuentra situado en el límite de tres importantes placas tectónicas que interactúan entre sí: la placa de Nazca, la placa del Caribe y la placa suramericana. A lo largo de la historia geológica, la interacción de estas tres placas dio origen al sistema orogénico de los Andes del Norte -compuesto por distintas cadenas de montaña separadas por valles y depresiones intramontañosas-, a deformaciones en la corteza continental, a la actividad volcánica y a una intensa actividad sísmica en el territorio colombiano. La placa de Nazca converge hacia el este con respecto a la telúricos naturales de la tierra. Cuando estas son de alta intensidad, pueden ocasionar daños en las zonas rurales o urbanas. Se clasifican en ondas de cuerpo y ondas superficiales (Quevedo, Pérez y Senmache, 2010).

Para calcular el tiempo de propagación de la onda $\mathrm{P}$, se debe considerar la estructura 3D de la tierra o usar un modelo regional ya establecido. Se determina primero una ubicación preliminar en la superficie, a partir de una triangulación con la participación de un mínimo de tres estaciones sísmicas. Cuando se produce un evento sísmico, las ondas P y S generadas se desplazan en todas las direcciones por sus características de propagación y el medio por el que se propagan. Estas ondas tienen velocidades diferentes -las ondas $P$ son más veloces que las ondas S- (Keiiti, 2002).

placa Sudamericana a una velocidad relativa aproximada de $7 \mathrm{~cm} /$ año (Servicio Geológico Colombiano, 2017).

La convergencia entre las dos placas se absorbe entre la zona de subducción del Pacífico a lo largo de los sistemas de fallas y pliegues activos en las tres cordilleras y valles de la cadena andina colombiana. La placa del Caribe, a su vez, converge con la placa suramericana a una velocidad entre 1 y $2 \mathrm{~cm}$ por año. La actividad sísmica de estas dos placas no es contundente, debido a que la subducción es incipiente.

La interacción de estas tres placas tectónicas produce una serie de fallas dentro del territorio colombiano en dirección SE-NW, como la falla de Romedal, Cauca-Patía, Soapaga, el borde 


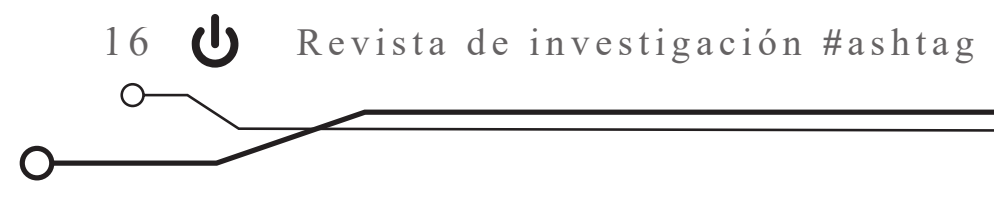

de la cordillera oriental, Bucaramanga-Santa Marta, entre otras. El sistema de fallas de Romedal es uno de los más importantes del país y de Sudamérica: se extiende desde Barranquilla (Colombia) hasta Talara (Perú). Su principal característica es la de presentar alta probabilidad de actividad sísmica superficial, con terremotos $M L \leq 4.0$ y una sismicidad intermedia con terremotos $M L \geq 5.0$; históricamente, se han presentado sismos superiores a $M L=6.0$ en la región de la Sierra Nevada de Santa Marta.

El sistema del piedemonte llanero conforma uno de los sistemas de fallas más activos de los Andes en el norte de Sudamérica: representa una posible fuente sismogénica de alta destrucción en ciudades como Bogotá y Villavicencio, con sismos de una magnitud de $M_{\mathrm{w}}=6.5$. La región se caracteriza por tener una alta actividad sísmica con una magnitud variada y de profundidades

\section{Sismómetro}

Es un instrumento empleado para medir los movimientos generados por la Tierra. Está basado en el principio de inercia, cuyo fundamento consiste en que los objetos tienden a resistirse al movimiento o a variar su velocidad. Debido a esta causa, el movimiento del suelo puede ser medido respecto a una masa que se encuentre suspendida. Este mecanismo usualmente se compone de un sistema masa-resorte que va unido a un soporte acoplado al suelo (Keiiti, 2002).

Al momento de un evento sísmico, el sistema se estimula por acción de las ondas generadas. focales de hasta unos $60 \mathrm{~km}$, que son asociables a la actividad de esta zona de fallas. Además, el análisis de los principales rasgos geomorfológicos señala alta actividad neotectónica, lo que indica que el proceso orogénico en la cordillera no ha cesado (Hernández, 2015).

El nido de Bucaramanga corresponde a una de las zonas sismogénicas más importantes de la Tierra. El nido apareció como consecuencia de la concentración de esfuerzos causados por la convergencia entre las placas Caribe, Nazca y sudamericana. La zona de mayor actividad sísmica en Santander está limitada a un área relativamente pequeña, cerca de unos $10000 \mathrm{~km}^{2}$. Solo en el lapso entre 1964 y 1973, la oficina para la localización de epicentros en Estados Unidos informó de 189 terremotos con epicentros en esta región, con una profundidad de entre 140 y 175 $\mathrm{km}$ y magnitudes mayores o iguales a $M_{\mathrm{w}}=4.2$.

La masa se mantiene un instante en reposo y cuando logra salir de este estado permite observar el movimiento del suelo. Las ondas se grafican en un cilindro de papel que se encuentra girando de manera constante; para que estas mediciones puedan ser muy precisas, se necesita un medio para amortiguar la masa. Durante la historia se han desarrollado diferentes tipos de sismógrafos que han permitido observar y evaluar el comportamiento de los sismos. Estos pueden ser clasificados de acuerdo con su rango espectral (tabla 1). 
Tabla 1. Tipos de sismómetros

\begin{tabular}{lll} 
& \multicolumn{1}{c}{ Instrumento } & \multicolumn{1}{c}{ Rango espectral } \\
A & Piezoeléctricos & $103-105 \mathrm{~Hz}$ \\
B & Acelerómetros & $0,01-100 \mathrm{~Hz}$ \\
C & Geófonos de prospección & $4-1.500 \mathrm{~Hz}$ \\
D & Sismómetros de corto periodo & $0,2-2 \mathrm{Sg}$ \\
E & Sismómetros de largo periodo & $10-100 \mathrm{Sg}$ \\
F & Extensómetros, deformímetros, distanciómetros & $\mathrm{DC}-0,5 \mathrm{~Hz}$ \\
G & Banda ancha & $0,003-30 \mathrm{~Hz}$ \\
\hline
\end{tabular}

Fuente: elaboración propia

\section{Desarrollos y tendencias en sismología}

La investigación titulada "Sistema distribuido de detección de sismos usando una red de sensores inalámbrica para alerta temprana" busca la detección de eventos perturbadores que utilizan sensores COTS, como los incorporados en los teléfonos inteligentes, cuya aplicación es de bajo consumo de energía. El estudio plantea la utilización del sensor inercial de los teléfonos inteligentes como un acelerógrafo y aprovechar los sistemas que optimizan los cálculos distribuidos en estos aparatos, así como sus capacidades de comunicación e integración, con el fin de proporcionar tiempo adicional para la alerta temprana en situaciones de desastre (Zambrano et al., 2015).

Los científicos de la Sociedad Sismológica de América implementan centros de investigación y monitoreo de sismos, donde las ondas de cuerpo-terremoto locales y regionales son registradas por la red sísmica mediante caminos fuente-estación. Los datos se transmiten a la red mencionada tras seleccionar la región del epicentro del terremoto, para analizar el decaimiento de la amplitud espectral de las ondas de cuerpo con la distancia al hipocentro, cuyas curvas de atenuación obtenidas indican las amplitudes espectrales (Castro et al., 2008).

Investigadores del Instituto de Ciencias de la Tierra del Consejo Superior de Investigaciones Científicas (CSIC) registraron microtemblores generados por la circulación del metro, el tráfico, conciertos y múltiples vibraciones de diversos orígenes, gracias un sismómetro instalado en Barcelona. En esta investigación, se destaca el papel que puede tener la sismología como herramienta adjunta al monitoreo de fenómenos relacionados a la actividad humana que tienen un impacto significativo en el ambiente. De igual manera, también podrá participar en la determinación de los niveles de tráfico de un sector, monitorear la estabilidad estructural de una edificación con el paso de los años y de acuerdo con el entorno donde se construyó o las modificaciones urbanísticas que ha sufrido, y analizar el comportamiento y rendimiento estructural de los edificios empleados en distintas actividades 


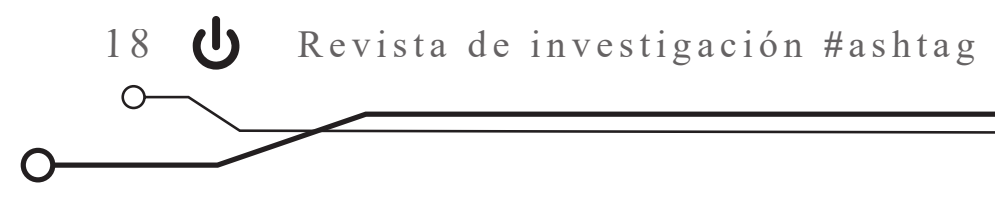

humanas, como conciertos y eventos deportivos (Díaz et al., 2017).

Central Nervous System for the Earth (CENSE), lo que en español se traduce a Sistema Nervioso Central para la Tierra, es un proyecto de HP Labs. Consiste en una red ultrainteligente de millones de sensores a nanoescala diseñados para sentir, saborear, oler, ver y escuchar lo que está sucediendo el mundo. Cuando el proyecto esté implementado en su totalidad, tendrá la capacidad de recopilar datos muy rápidamente y transmitirlos a potentes motores de computación que analizarán y actuarán sobre la información en tiempo real. De esta manera, sus redes estarán destinadas a mejorar la forma en que los gobiernos, las empresas y la sociedad responden y gestionan los cambios ambientales, biológicos, físicos y estructurales. Dentro del proyecto CENSE, se está desarrollando un acelerómetro que será mil veces más sensible que los que se producen actualmente -por ejemplo, permitiría notar cambios en las vibraciones por fuera de la Tierra-. Será instalado en una cápsula artificial en el fondo del mar (Wylie, 2009).

Ingenieros del departamento de Ingeniería Civil de la Universidad Nacional de Ciencia

\section{Conclusiones}

Parte importante y necesaria en las acciones de prevención de desastres y la gestión de riesgos es el monitoreo de los fenómenos naturales y los sistemas de alerta temprana. Las redes de monitoreo, por un lado, permiten obtener información necesaria para comprender y mejorar el conocimiento científico de amenazas y riesgos, punto de partida para la implementación de políticas efectivas de prevención y mitigación. Por y Tecnología de Taiwán han estado desarrollando un acelerómetro basado en un sistema microelectromecánico llamado P-Alert, de bajo costo. La finalidad del dispositivo es evaluar la seguridad en las construcciones después de un terremoto. La construcción se somete a una serie de pruebas de sacudida y se utilizan las curvas de fragilidad de diferentes niveles de daño junto al cálculo de las aceleraciones para medir el daño potencial y estimar el nivel de avería aceptable en los edificios (Hsu et al., 2018).

El instituto de semiconductores de la Academia China de Ciencias, ubicada en Beijing, desarrolló un sismómetro de fibra óptica en un resonador de banda ancha FBG (fiber bragg grating). La longitud de onda de ultrabaja frecuencia de alta resolución de barrido láser permitió que el sismómetro funcionara en un rango de los $0,01 \mathrm{~Hz}$ a los $10 \mathrm{~Hz}$, con una resolución de longitud de onda de 330 p/g. Emplea un método de correlación de tres de canales. Mediante pruebas se lograron registrar microsismos y sismos muy distantes. Es la primera vez que un sismómetro de periodo medio basado en FBG logra obtener registros muy exactos sin interferencia del ruido propio NHNM (new high noise model) y rango dinámico de 100 Db (Huang et al., 2018).

otro lado, diferentes instrumentos y tecnologías empleadas en el monitoreo y vigilancia de los fenómenos permiten detectar, dar seguimiento $\mathrm{y}$ pronosticar las amenazas naturales que puedan impactar a la población.

Por lo tanto, los sistemas de monitoreo contribuyen a la toma efectiva de decisiones ante situaciones de riesgo y permiten alertar de manera 
oportuna a la población. De esta manera, los sistemas de alerta tienen como objetivo facultar a las personas y a las comunidades que se encuentren en peligro para actuar con tiempo suficiente y de manera adecuada. De este modo, sería posible minimizar los daños personales, evitar la pérdida de vidas y reducir los daños a las propiedades y el medio ambiente.

\section{Referencias}

BBC Mundo. (13 de enero del 2010). Terremoto devasta Haití. Recuperado de http://www.bbc. com/mundo/internacional/2010/01/100112_2231_terremoto_haiti_irm.shtml

Castro, R., Condori, C., Romero, O., Jacques, C. y Suter, M. (2008). Seismic Attenuation in Northeastern Sonora, Mexico. Bulletin of the Seismological Society of America. 98(2), 722-732. Recuperado de https://pubs.geoscienceworld.org/ssa/bssa/article-abstract/98/2/722/341890/ Seismic-Attenuation-in-Northeastern-Sonora

Díaz, J., Ruíz, M. Sánchez-Pastor, P. y Romero, P. (2017). Urban Seismology: On the origin of earth vibrations within a city. Scientific Reports, 7, 1-11. Recuperado de https://www.nature. com/articles/s41598-017-15499-y.pdf

El Tiempo. (25 de enero de 1999). Terremotos en la historia. Recuperado de https://www.eltiempo.com/archivo/documento/MAM-892704

Gómez, M. (18 de septiembre del 2017). 10 terremotos quecambiaron la historia. Eltiempo.es. Recuperado de https:/ / noticias.eltiempo.es/los-10-terremotos-mas-importantes-de-la-historia/

Hernández, O. (2015). Evaluación de amenaza sísmica en municipios del departamento de Cundinamarca [tesis de grado]. Universidad Distrital Francisco José de Caldas, Bogotá, Colombia.

Hsu, T., Yin, R. y Wu, Y. (2018). Evaluating Post-Earthquake Building Safety Using Economical mEMs Seismometers. Sensors, 18(5), 1-12. Recuperado de https://www.mdpi. com/1424-8220/18/5/1437/htm

Huang, W., Zhang, W., Luo, Y. Li, L., Liu, W. y Li, F. (2018). Broadband fBG Resonator Seismometer: Principle, Key Technique, Self-Noise, and Seismic Response Analysis. Optics Express, 26(8), 10705-10715. Recuperado de https://www.osapublishing.org/oe/fulltext. cfm?uri $=$ oe-26-8-10705\&id $=385568$

Keiiti, P. (2002). Quantitative Seismology. Sausalito, CA: University Science Books.

Quevedo, J., Pérez, C. y Senmache, J. (2010). Ondas sísmicas [documento interno de trabajo]. Universidad Católica Santo Toribio de Mogrovejo, Chiclayo, Perú. 


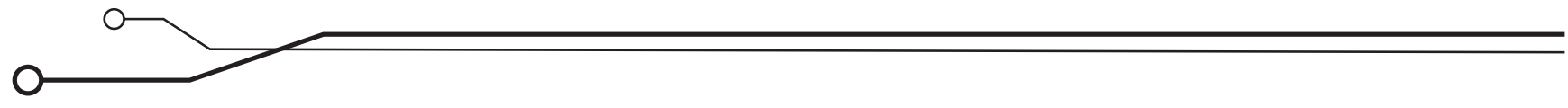

Servicio Geológico Colombiano (SGC). (2017). Conceptos básicos [recurso en línea]. Recuperado de http://200.119.88.135/RSNC/index.php/material-educativo/conceptos-basicos

Servicio Geológico Colombiano (sGC). (2018). Red Sismológica Nacional de Colombia [recurso en línea]. Recuperado de https://www2.sgc.gov.co/Paginas/servicio-geologico-colombiano. aspx

Tarbuck, E. y Lutgens, F. (2005). Ciencias de la tierra (8 ${ }^{\text {va }}$ ed.). Madrid: Pearson Educación.

Wylie, M. (2009). Earth Calling: Turn off the Lights [recurso en línea]. Hewlett Packard. Recuperado de http://www.hpl.hp.com/news/2009/oct-dec/cense.html

Zambrano, A., Pérez, I., Palau, C. y Domingo, M. (2015). Sistema distribuido de detección de sismos usando una red de sensores inalámbrica para alerta temprana. Revista Iberoamericana de Automática e Informática Industrial, 12(3), 260-269. Recuperado de https://polipapers. upv.es/index.php/RIAI/article/view/9360 